Rev. Int. Contam. Ambie. 36 (2) 321-331, 2020

DOI: $10.20937 /$ RICA.53402

\title{
ANÁLISIS DEL CONTENIDO DE BENCENO EN LAS GASOLINAS Y ESTIMACIÓN DE EMISIONES DE ESTE COMPUESTO AL AMBIENTE
}

Analysis of benzene content in gasolines and emissions estimation of this compound to the environment

Fabiola Selene ALCÁNTAR GONZÁLEZ, Rodrigo ELIZALDE SEGOVIA, María Guadalupe OLVERA SANTOS, Daniel LÓPEZ HERRERA y M. Javier CRUZ GÓMEZ*

Departamento de Ingeniería Química, Facultad de Química, Universidad Nacional Autónoma de México. Av. Universidad 3000, 04510 Ciudad de México, México

*Autor para correspondencia: mjcg@unam.mx

(Recibido: septiembre 2018; aceptado: octubre 2019)

Palabras clave: normatividad ambiental, gasolineras, caracterización, CdMx

\section{RESUMEN}

El uso de gasolinas es un tema de gran interés en el ámbito ecológico debido a las emisiones que generan los automotores, por lo que el control de los parámetros que regulan la calidad de las gasolinas es necesario para mantener una buena calidad del aire. La Ciudad de México (CdMx) es una metrópoli con una flota vehicular registrada de 4421797 (2014), factor que influye en la calidad del aire. Las especificaciones de calidad de la gasolina en México están reguladas por la NOM-016-CRE-2016. Uno de los compuestos regulados por esta norma es el benceno, que se considera un agente carcinogénico. Se ha determinado que la presencia de benceno en la gasolina puede afectar la salud de la población, ya que al ser un compuesto volátil se evapora con gran facilidad, poniéndose en contacto con la atmósfera y por consecuencia con la población. En este trabajo se cuantificó la cantidad de benceno presente en las gasolinas que se comercializan en la CdMx mediante el uso de la técnica ASTM D5769-15 y se determinó que el contenido promedio de este compuesto fue de $0.361 \%$ para la gasolina Magna, mientras que el contenido promedio de benceno en las gasolinas importadas fue de $0.55 \%$. En cuanto a las gasolinas producidas en el Sistema Nacional de Refinación, el contenido de benceno fue de $1.288 \%$, valor superior al de las gasolinas importadas y comercializadas en la CdMx. Asimismo, se estimó una emisión de 354.79 t de benceno durante 2017 debido al uso de automóviles en la CdMx.

Key words: environmental laws, gas stations, characterization, Mexico City

\begin{abstract}
Use of gasolines has a primordial interest in the ecological area, due to the emissions generated by combustion engines. In order to maintain a good air quality, it is necessary to control several gasoline quality parameters. Mexico City is a metropolis with 4421797 vehicles registered in 2014, therefore air quality problems are constantly present. Mexican gasoline quality specifications are regulated by Mexican Official Standard NOM-016-CRE-2016. Benzene, one of the compounds regulated by NOM016-CRE-2016, is known to be a carcinogenic agent. Moreover, it is considered that the presence of benzene in gasoline can affect population health due to its volatility,
\end{abstract}


which gets it to be in contact with the atmosphere and consequently with the population. In this work, the average amount of benzene present in gasoline commercialized in Mexico City was quantified by ASTM D5769-15. Results showed it was $0.361 \%$ in Magna gasoline, whereas the average amount of benzene present in imported gasoline was $0.55 \%$ and $1.28 \%$ in the gasoline produced locally by PEMEX National Refineries System. This value was higher than imported and commercialized gasoline. In addition, the estimation of benzene emissions by mobile sources was $354.79 \mathrm{t}$ during 2017.

\section{INTRODUCCIÓN}

En las últimas décadas el dinamismo demográfico mundial ha generado regiones urbanas de gran extensión. Muchas de estas grandes ciudades requieren diversos tipos de servicios y soluciones para afrontar los problemas causados por tener una gran cantidad de habitantes en una misma zona. El impacto de la contaminación en estas ciudades afecta a los pobladores, la flora, la fauna, los suelos, el agua y la calidad del aire. La Ciudad de México (CdMx) es una de estas megaciudades, y muchas de las dificultades que afronta día con día están relacionadas con la contaminación (Baklanov et al. 2016).

Mediante el análisis de la calidad del aire se han podido identificar los principales contaminantes que afectan a la troposfera: ozono $\left(\mathrm{O}_{3}\right)$, partículas en suspensión (PM, por sus siglas en inglés), óxidos de azufre $\left(\mathrm{SO}_{\mathrm{x}}\right)$, óxidos de nitrógeno $\left(\mathrm{NO}_{\mathrm{x}}\right)$ y compuestos orgánicos volátiles (VOC, por sus siglas en inglés). Además, se ha identificado que los mayores niveles de contaminación se presentan en marzo y mayo, así como en la zona norte-oriente de la CdMx. De igual forma, se ha reportado una disminución anual de los contaminantes del aire a partir de 2000 en la Zona Metropolitana del Valle de México (ZMVM) (Calderón-Garcidueñas et al. 2015, Meraz et al. 2015).

Sin embargo, a pesar de los esfuerzos realizados, muchos de estos contaminantes siguen presentes en el aire de la ciudad y se han podido identificar los ciclos anuales de su generación (Meraz et al. 2015), por lo que se considera que la solución al problema de la calidad del aire de la CdMx requiere de un extenso y persistente programa de regulación que afronte la contaminación del aire, contemplando sus efectos sobre la salud, el transporte y la administración de la ciudad (Molina y Molina 2004).

En la CdMx se ha implementado un monitoreo de contaminantes del aire por medio de múltiples estaciones de medición localizadas en diferentes puntos de la ciudad. Estos datos se obtienen cada hora durante los 365 días del año, por medio de índices que emplean promedios horarios de los contaminantes en el aire. Así se obtiene el índice de contaminación conocido como Índice Metropolitano de la Calidad del Aire (IMECA), que nos permite conocer el nivel de contaminación del aire y alerta a la población de la CdMx en caso de contingencia.

Con relación al consumo de petrolíferos, en el Inventario de Emisiones de la CdMx se reportó que durante 2014 se consumieron 543 petajulios (PJ) en la ZMVM, de los cuales 197 PJ corresponden al uso de gasolina Magna, 39.5 PJ a la gasolina Premium, 70.4 PJ al diésel, 95 PJ a gas L.P. y 141.1 PJ a gas natural. Este consumo de petrolíferos tuvo una contribución del $58 \%$ del transporte, $26 \%$ de la industria y $13 \%$ del sector habitacional. Cabe destacar que en el caso del transporte se consumieron 7.32 millones de $\mathrm{m}^{3}$ de gasolina, de los cuales 6.1 millones de $\mathrm{m}^{3}$ correspondieron a gasolina Magna y 1.2 millones a gasolina Premium (SEDEMA 2016).

En México, la Norma Oficial Mexicana que rige la calidad de los combustibles es la NOM016-CRE-2016. Especificaciones de calidad de los petrolíferos (CRE 2016), expedida por la Comisión Reguladora de Energía. Esta NOM remplaza a la norma de emergencia NOM-EM-005-CRE-2015 (CRE 2015), cuyo antecedente es la NOM-086-SEMARNAT-SENER-SCFI-2005 (SEMARNAT 2006) que estuvo vigente hasta 2015.

Por otra parte, se han realizado estudios en los cuales se demuestra que la presencia de benceno en las gasolinas puede afectar la salud de la población, ya que al ser un compuesto volátil se evapora con gran facilidad, entrando en contacto con la atmósfera y por consiguiente con la población. Un efecto a corto plazo que se ha observado en personas expuestas a concentraciones de $1 \mathrm{ppm}$ de benceno en el aire es la disminución de glóbulos blancos, plaquetas y otros índices hematológicos (Johnson et al. 2007). Además, la exposición a benceno a bajas concentraciones ha causado efectos genotóxicos como mutación de ADN, anormalidades cromosómicas y daño oxidativo. La exposición a largo plazo puede contribuir al desarrollo de carcinogenicidad (Moro et al. 2017), ya 
que el benceno pertenece al grupo 1 (agente carcinogénico) según la clasificación de la Agencia Internacional para la Investigación sobre el Cáncer (IARC). De acuerdo con estudios realizados por Moro et al. (2017) se ha determinado que hay mayor velocidad de biotransformación de benceno hacia ácido trans, trans-mucónico (metabolito del benceno) en orina en las mujeres, debido a que metabolizan del 23 al $26 \%$ más benceno que los hombres, aun cuando estén expuestos a los mismos niveles de este compuesto (Moro et al. 2017).

Debido a los efectos negativos de las concentraciones de benceno presente en el aire para la salud, la contribución de este trabajo consiste en determinar el contenido de benceno en las gasolinas que se comercializan en la CdMx y compararlo con las gasolinas importadas y con las producidas en el Sistema Nacional de Refinación (SNR).

\section{MATERIALES Y MÉTODOS}

\section{Muestreo}

Se consideraron el número, la localización y los requerimientos para obtener la franquicia de las gasolineras establecidas en la CdMx (Torres 2016), utilizándose la Guía de Pemex como herramienta de apoyo (PEMEX 2017). Así, se realizó un muestreo estratificado de asignación proporcional de las 398 gasolineras establecidas en las 16 alcaldías de la $\mathrm{CdMx}$, donde se muestrearon 100 gasolineras (Fig. 1). En el muestreo se consideró la cantidad de gasolineras presentes en cada alcaldía, ya que éstas no cuentan con el mismo número de gasolineras; por lo mismo, se decidió seleccionar proporcionalmente el número de gasolineras (Cuadro I). La recolección de muestras de gasolina se llevó a cabo en frascos herméticos de boquilla ancha de $500 \mathrm{~mL}$ para la gasolina Magna y Premium. Se recolectaron un total de 100 muestras de gasolina Magna y 100 muestras de gasolina Premium. Para realizar la inyección de las muestras en el GC/MS se ocuparon 200 viales de $1.5 \mathrm{~mL}$.

\section{Cuantificación de benceno}

Se empleó el método ASTM D5769-15 (Determinación de benceno, tolueno y total de compuestos aromáticos en gasolina refinada mediante cromatografía de gases- espectrometría de masas), para lo cual se utilizó un cromatógrafo de gases 7890B acoplado a un espectrómetro de masas 5977A y un inyector automático 7693A, así como una columna de dimetilpolisiloxano de $60 \mathrm{~m} \times 0.25 \mathrm{~mm}$ de diámetro

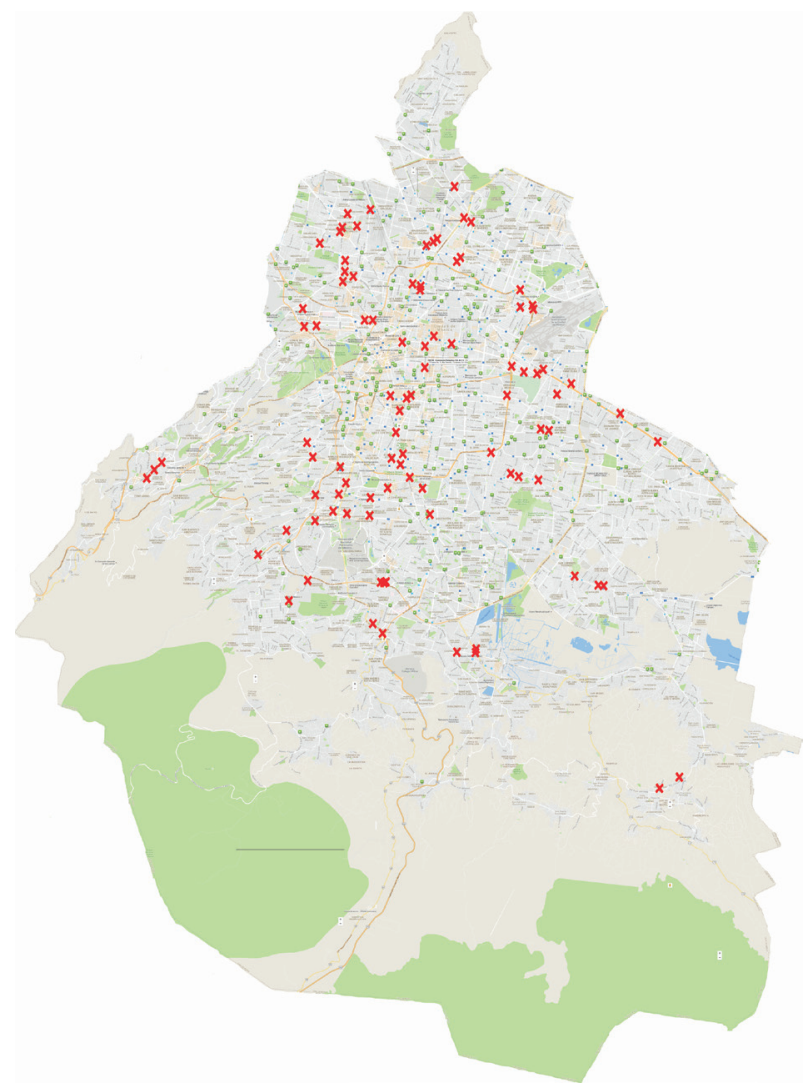

Fig. 1. Imagen de la $\mathrm{CdMx}$ con las 100 gasolineras seleccionadas para el muestreo representativo

CUADRO I. NÚMERO TOTAL DE GASOLINERAS EN LA CdMx POR ALCALDÍA Y GASOLINERAS SELECCIONADAS PARA EL MUESTREO PROPORCIONAL

\begin{tabular}{lcc}
\hline Alcaldía & $\begin{array}{c}\text { Total de } \\
\text { gasolineras }\end{array}$ & $\begin{array}{c}\text { Gasolineras } \\
\text { seleccionadas }\end{array}$ \\
\hline Álvaro Obregón & 28 & 8 \\
Azcapotzalco & 22 & 8 \\
Benito Juárez & 39 & 8 \\
Coyoacán & 29 & 8 \\
Cuajimalpa Morelos & 7 & 3 \\
Cuauhtémoc & 46 & 8 \\
Gustavo A. Madero & 46 & 8 \\
Iztacalco & 12 & 8 \\
Iztapalapa & 62 & 8 \\
Magdalena Contreras & 2 & 2 \\
Miguel Hidalgo & 31 & 8 \\
Milpa Alta & 2 & 2 \\
Tláhuac & 7 & 3 \\
Tlalpan & 23 & 7 \\
Venustiano Carranza & 33 & 8 \\
Xochimilco & 9 & 3 \\
Total & 398 & 100 \\
\hline
\end{tabular}


interno y $1.0 \mu \mathrm{m}$ de diámetro de capa, de Agilent Technologies (California, Estados Unidos). Se aplicaron las condiciones 1 establecidas en el método.

Además, se utilizó un paquete de estándares con cinco muestras a diferentes concentraciones de compuestos aromáticos de gasolina del proveedor Acccustandard (New Haven, USA) para obtener la curva de calibración o patrón utilizada como referencia en la medición cuantitativa del porcentaje en masa de benceno presente en las muestras de gasolina. Se determinó la densidad de las muestras de gasolina con un picnómetro, considerando la temperatura a la que se realizó la medición (rango de 18 a $25^{\circ} \mathrm{C}$ ); posteriormente se determinó el porcentaje en volumen de benceno en la gasolina de acuerdo con la ecuación 1 (ASTM D5769):

$\% v=\% \frac{m_{i}}{m_{t}} *\left(\frac{D_{f}}{D_{b}}\right)$

donde $\frac{m_{i}}{m_{t}}$ es el porcentaje de la relación de la masa del benceno respecto del total de la muestra de gasolina; $D_{\mathrm{f}}$ es la densidad de la gasolina, y $D_{\mathrm{b}}$ es la densidad del benceno.

\section{Simulación de emisiones en fuentes móviles}

Se realizó la estimación de emisiones de benceno provenientes del uso de automóviles, así como de los procesos de combustión y evaporación, mediante el uso del programa MOVES14a (Motor Vehicle Emision Simulator) de la Agencia de Protección del Medio Ambiente de Estados Unidos (USEPA, por sus siglas en inglés), ya que el programa está diseñado para estimar la cantidad de contaminantes que emiten los vehículos automotores. Dicha versión fue adaptada del modelo recomendado por la USEPA y la Secretaría de Medio Ambiente y Recursos Naturales (SEMARNAT), denominado MOVES-México, para utilizarse en inventarios de emisiones de la CdMx (SEDEMA 2016), ya que puede incluir datos de flota vehicular, composición del combustible, estándares de emisión vehicular, velocidades vehiculares promedio y condiciones locales de altitud y temperatura ambiente para México, además de tener la facilidad de hacer la simulación por estado, en este caso para la CdMx.

En el cuadro II se indican algunos parámetros que se consideraron en la simulación para la estimación de emisiones de benceno. La simulación se realizó considerando el mes de febrero de 2017, ya que en este periodo se llevó a cabo el muestreo, y la gasolina Magna, ya que su consumo es alrededor de cuatro veces mayor que el consumo de gasolina Premium en la CdMx (SEDEMA 2016). Asimismo, se consideró el número de automóviles registrados (Cuadro III) en las 16 alcaldías de la CdMx durante el 2014 por la Secretaría de Transporte (INEGI 2017) y se recopilaron las temperaturas, así como la humedad relativa para cada hora del día en los 28 días de febrero de 2017 para cada una de las alcaldías, ya que son datos requeridos por el software, obtenidos de las diferentes estaciones del Sistema de Monitoreo Atmosférico (SIMAT). Además, los datos de actividad de los automóviles se supusieron en kilómetros recorridos por día, de acuerdo con el inventario de emisiones de la CdMx de 2012 (SEDEMA 2013).

CUADRO II. ESPECIFICACIONES DEL PROGRAMA MOVES 14A PARA LA ESTIMACIÓN DE EMISIONES DE BENCENO

\begin{tabular}{ll}
\hline Parámetro & Especificación \\
\hline Motor & Motor de combustión interna \\
\hline Combustible & Gasolina Magna \\
\hline Tipo de camino & Mayormente urbano \\
\hline Composición & $\begin{array}{l}\text { Valor promedio del contenido de benceno } \\
\text { en gasolina Magna para cada delegación } \\
\text { determinado experimentalmente }\end{array}$ \\
\hline
\end{tabular}

CUADRO III. AUTOMÓVILES REGISTRADOS EN LA CdMx DURANTE 2014

\begin{tabular}{llc}
\hline Clave & Nombre & Automóviles \\
\hline 09002 & Azcapotzalco & 246116 \\
09003 & Coyoacán & 384193 \\
09004 & Cuajimalpa de Morelos & 118074 \\
09005 & Gustavo A. Madero & 471512 \\
09006 & Iztacalco & 199913 \\
09007 & Iztapalapa & 567836 \\
09008 & La Magdalena Contreras & 105423 \\
09009 & Milpa Alta & 45012 \\
09010 & Álvaro Obregón & 314817 \\
09011 & Tláhuac & 96530 \\
09012 & Tlalpan & 322547 \\
09013 & Xochimilco & 137709 \\
09014 & Benito Juárez & 391563 \\
09015 & Cuauhtémoc & 386246 \\
09016 & Miguel Hidalgo & 407837 \\
09017 & Venustiano Carranza & 226469 \\
09 & Ciudad de México & 4421797 \\
\hline
\end{tabular}

\section{RESULTADOS}

Se procedió a inyectar las muestras de gasolina en el cromatógrafo de gases 7890 B para obtener los 


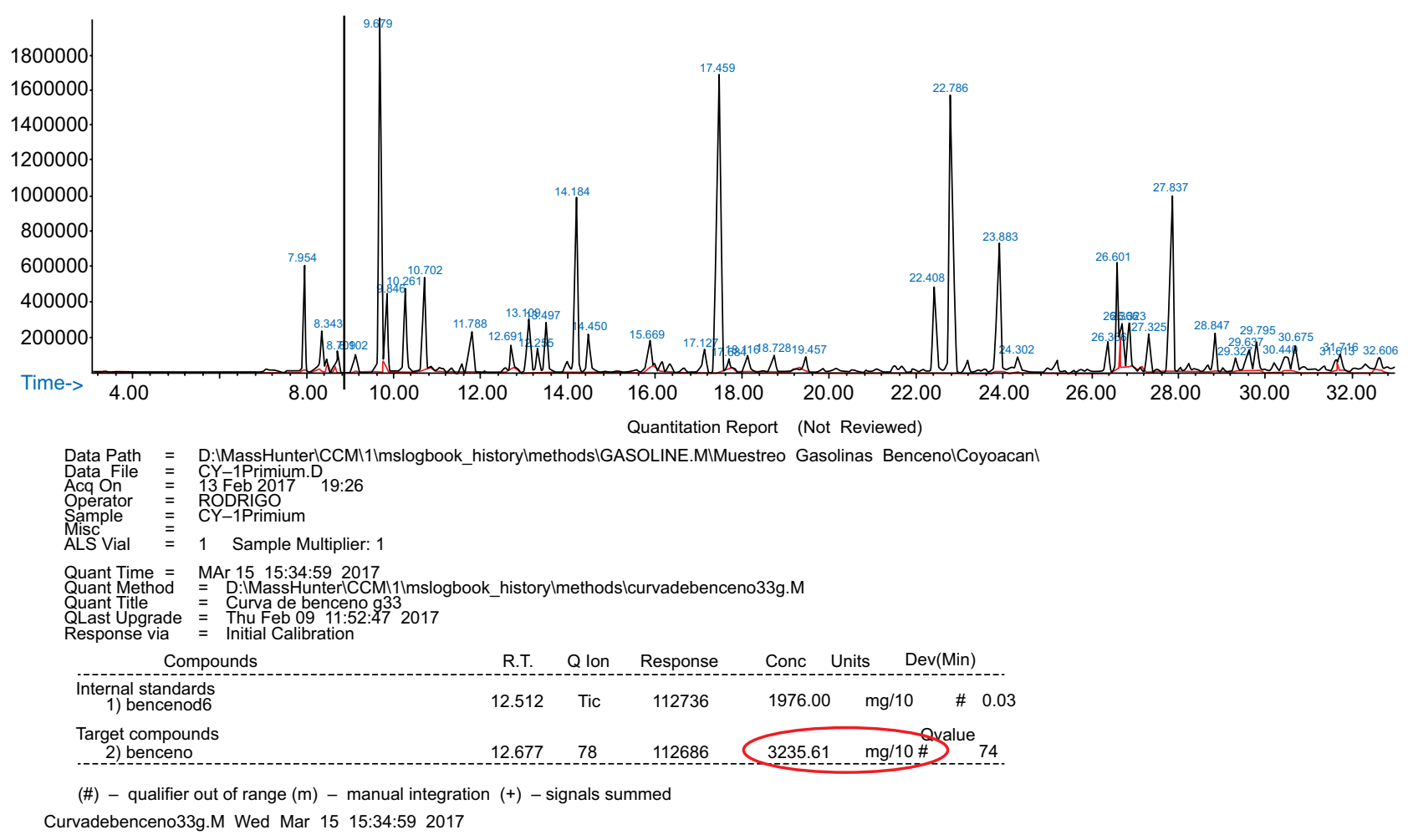

Fig. 2. Cromatograma de una muestra de gasolina para cuantificar el benceno

cromatogramas correspondientes, En la figura 2 se observa el cromatograma de la muestra de gasolina Premium en la alacaldía Coyoacán (CY-1). Se empleó la curva de calibración (Fig. 3) para obtener la relación de la masa del benceno respecto al total de muestra de gasolina. Después, considerando el valor promedio de las densidades de las gasolinas Magna y Premium de cada una de las alcaldías (Cuadro IV), se obtuvo el contenido promedio del porcentaje en

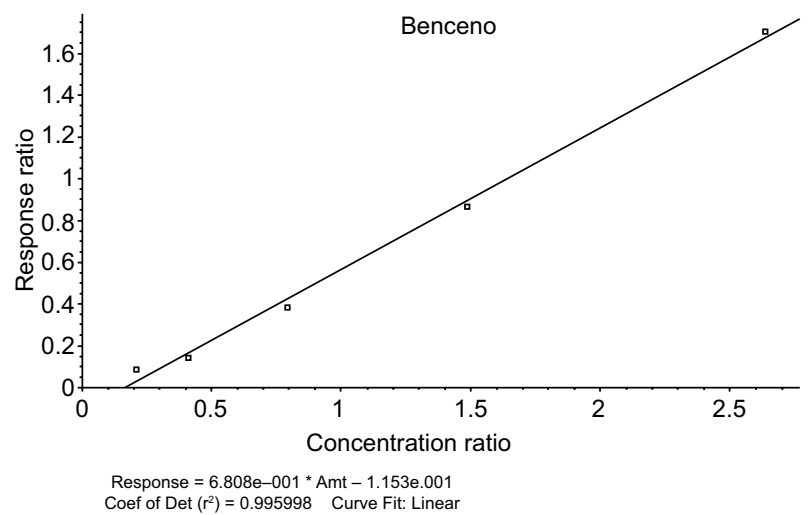

Fig. 3. Curva de calibración de benceno volumen del benceno (Fig. 4) para las 16 alcaldías de la CdMx durante febrero de 2017.

CUADRO IV. CONTENIDO PROMEDIO DE LADENSIDAD DE LAS GASOLINAS MUESTREADAS EN LA CdMx DURANTE FEBERERO DE 2017.

\begin{tabular}{lcc}
\hline & \multicolumn{2}{c}{ Densidad promedio } \\
\cline { 2 - 3 } Alcaldía & $\begin{array}{c}\text { Gasolina } \\
\text { Magna }(\mathrm{g} / \mathrm{mL})\end{array}$ & $\begin{array}{c}\text { Gasolina } \\
\text { Premium }(\mathrm{g} / \mathrm{mL})\end{array}$ \\
\hline Álvaro Obregón & 0.721 & 0.727 \\
Azcapotzalco & 0.721 & 0.728 \\
Benito Juárez & 0.728 & 0.728 \\
Coyoacán & 0.727 & 0.729 \\
Cuajimalpa De Morelos & 0.721 & 0.732 \\
Cuauhtémoc & 0.719 & 0.729 \\
Gustavo A. Madero & 0.725 & 0.729 \\
Iztacalco & 0.722 & 0.730 \\
Iztapalapa & 0.720 & 0.727 \\
La Magdalena Contreras & 0.721 & 0.727 \\
Miguel Hidalgo & 0.722 & 0.728 \\
Milpa Alta & 0.722 & 0.729 \\
Tláhuac & 0.721 & 0.728 \\
Tlalpan & 0.631 & 0.638 \\
Venustiano Carranza & 0.722 & 0.728 \\
Xochimilco & 0.721 & 0.729 \\
Promedio & 0.716 & 0.723 \\
\hline
\end{tabular}




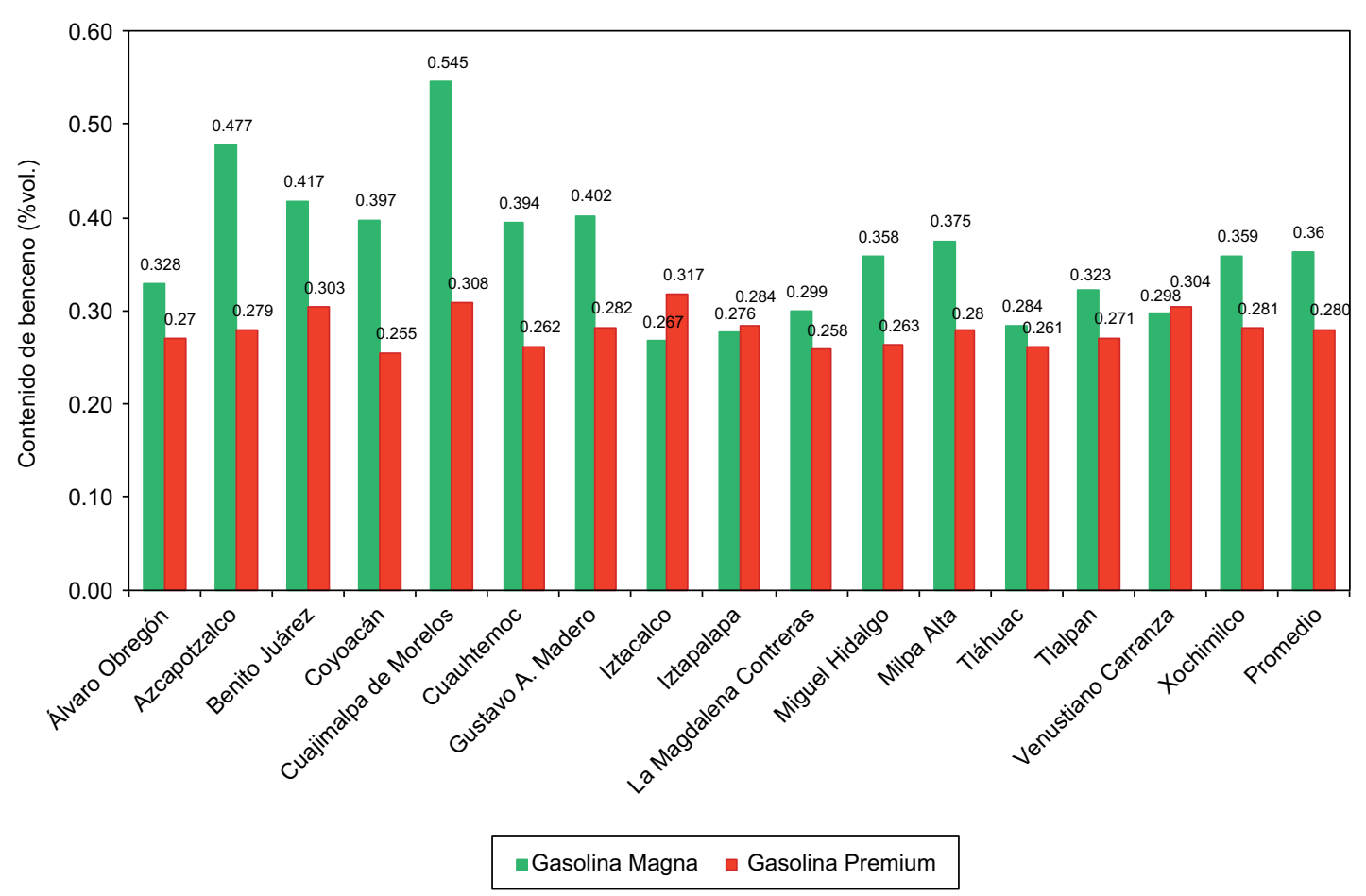

Fig. 4. Contenido promedio de benceno en las gasolinas para cada una de las alcaldías de la CdMx durante febrero de 2017

El Instituto Nacional de Acceso a la Información (INAI) proporcionó la información sobre el contenido de benceno en las gasolinas Magna y Premium producidas por el Sistema Nacional de Refinación (SNR) durante 2016 (Fig. 5). En la figura 6 se muestra el contenido de benceno de las gasolinas importadas en México durante el mismo año.

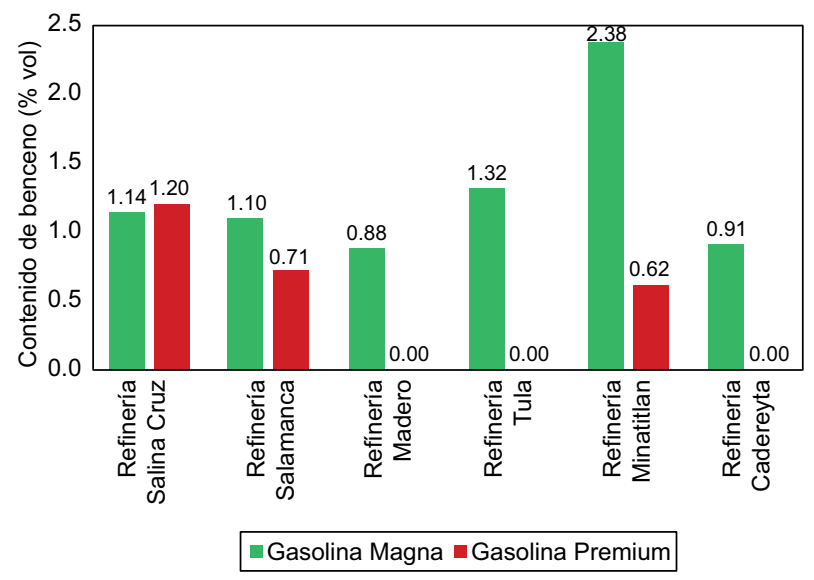

Fig. 5. Contenido promedio de benceno en las gasolinas producidas en el Sistema Nacional de Refinación durante 2016

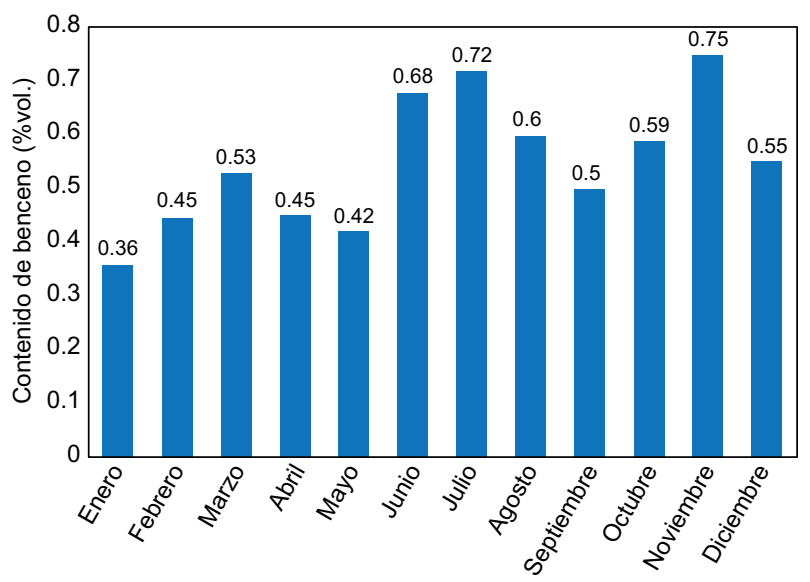

Fig. 6. Contenido de benceno en porcentaje en volumen de las gasolinas importadas durante 2016

\section{Estimación de emisiones en fuentes móviles}

En el cuadro $\mathbf{V}$ se muestran los resultados obtenidos de la estimación de emisiones de benceno por fuentes móviles utilizando el programa MOVES2014a, tomando en cuenta los 4421797 automóviles registrados en la CdMx durante 2014 (INEGI 2017). En la figura 7 se muestra la contribución de los procesos de combustión y evaporación simulados de 
CUADRO V. EMISIONES DE BENCENO POR AUTOMÓVILES DURANTE FEBRERO DE 2017

\begin{tabular}{lc}
\hline Alcaldía & Emisiones de benceno $(\mathrm{kg} / \mathrm{mes})$ \\
\hline Álvaro Obregón & 519.56 \\
Azcapotzalco & 3404.47 \\
Benito Juárez & 666.26 \\
Coyoacán & 649.28 \\
Cuajimalpa & 1740.26 \\
Cuauhtémoc & 673.81 \\
Gustavo A. Madero & 798.20 \\
Iztacalco & 5421.69 \\
Iztapalapa & 11223.09 \\
Magdalena Contreras & 2166.07 \\
Miguel Hidalgo & 680.08 \\
Milpa Alta & 446.46 \\
Tláhuac & 156.87 \\
Tlalpan & 531.38 \\
Venustiano Carranza & 369.84 \\
Xochimilco & 118.62 \\
Total & 29565.96 \\
\hline
\end{tabular}

los automóviles para estimar las emisiones de benceno procedentes del escape en movimiento, el arranque del escape, la liberación de vapores de combustión por venteo, la evaporación por permeación, la evaporación de combustible por fuga, el cigüeñal en movimiento y el arranque del cigüeñal.

En la figura 8 se presentan las emisiones de benceno por hora en las diferentes alcaldías de la CdMx.

\section{DISCUSIÓN}

Una de las principales fuentes de benceno en el aire es consecuencia de la emisión de vapor de la gasolina. Se estima que más del $60 \%$ del benceno en el aire se debe a la vaporización y a la incompleta combustión de la gasolina (Perry y Gee 1994, Wallace 1996), la cual puede variar dependiendo del contenido de benceno en la gasolina (Verma y des Tombe 2002).

Además, el reabastecimiento de combustible para automóviles en estaciones de gasolina, que es una importante fuente de exposición al benceno en el aire (superior a $10 \mathrm{ppm}$ ), puede ocurrir al menos una vez por semana por automóvil en ciudades industrializadas, lo cual indica que la exposición ambiental a benceno puede ocurrir varias veces durante el tiempo de vida de una persona (Egeghy et al. 2002).

Otras fuentes importantes de emisiones de benceno son las fuentes fijas tales como las plantas petroquímicas o refinerías. En un estudio realizado por la USEPA, concentraciones superiores de 0.2 ppm fueron registradas en comunidades de Elizabeth y Bayonne adyacentes a las plantas petroquímicas de Nueva Jersey (Wallace et al. 1987). La máxima concentración de benceno típica fue de 0.03 a 0.07 ppm con un nivel mínimo de 0.01 ppm en áreas lejanas a las plantas químicas, lo cual indica que la residencia cercana a plantas químicas puede incrementar el riesgo de exposición a niveles elevados de benceno. Asimismo, cuando las personas están expuestas a una concentración de 1 a 2 ppm de benceno en el aire por 40 años o más, se incrementa el riesgo de desarrollar cáncer hematológico (Johnson et al. 2007).

Por otra parte, de acuerdo con el Instituto Nacional de Ecología y Cambio Climático (INECC), se llevó a cabo una evaluación de las modificaciones a la NOM-016-CRE-2016 en la cual se menciona que la regulación americana conocida como Programa de

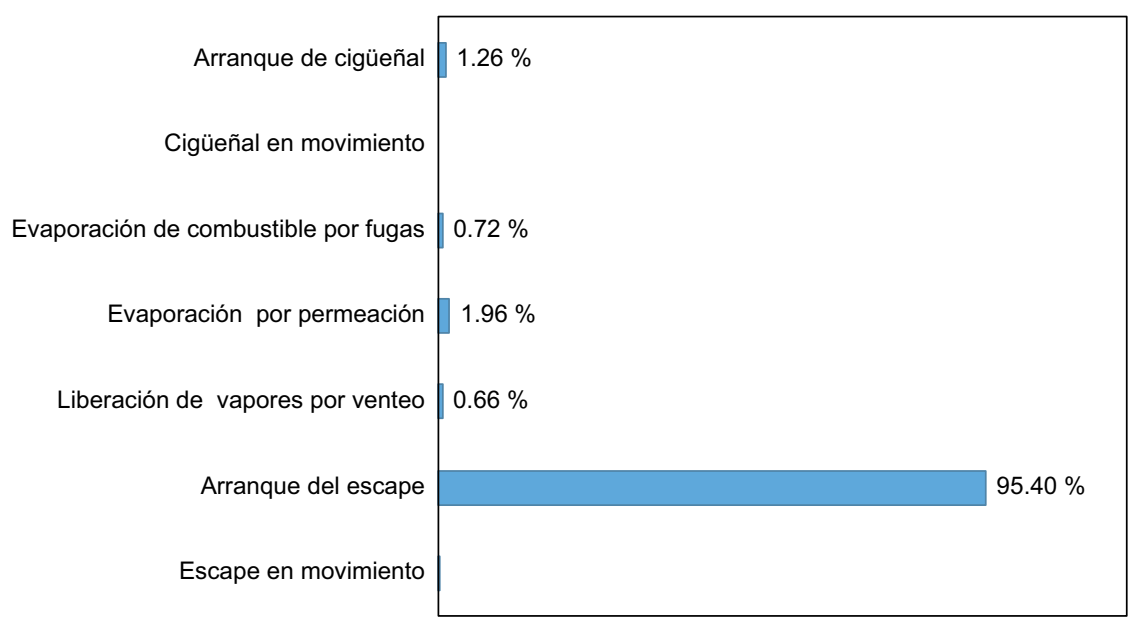

Fig. 7. Porcentaje de contribución de los procesos de combustión y evaporación simulados para la estimación de emisiones de benceno 


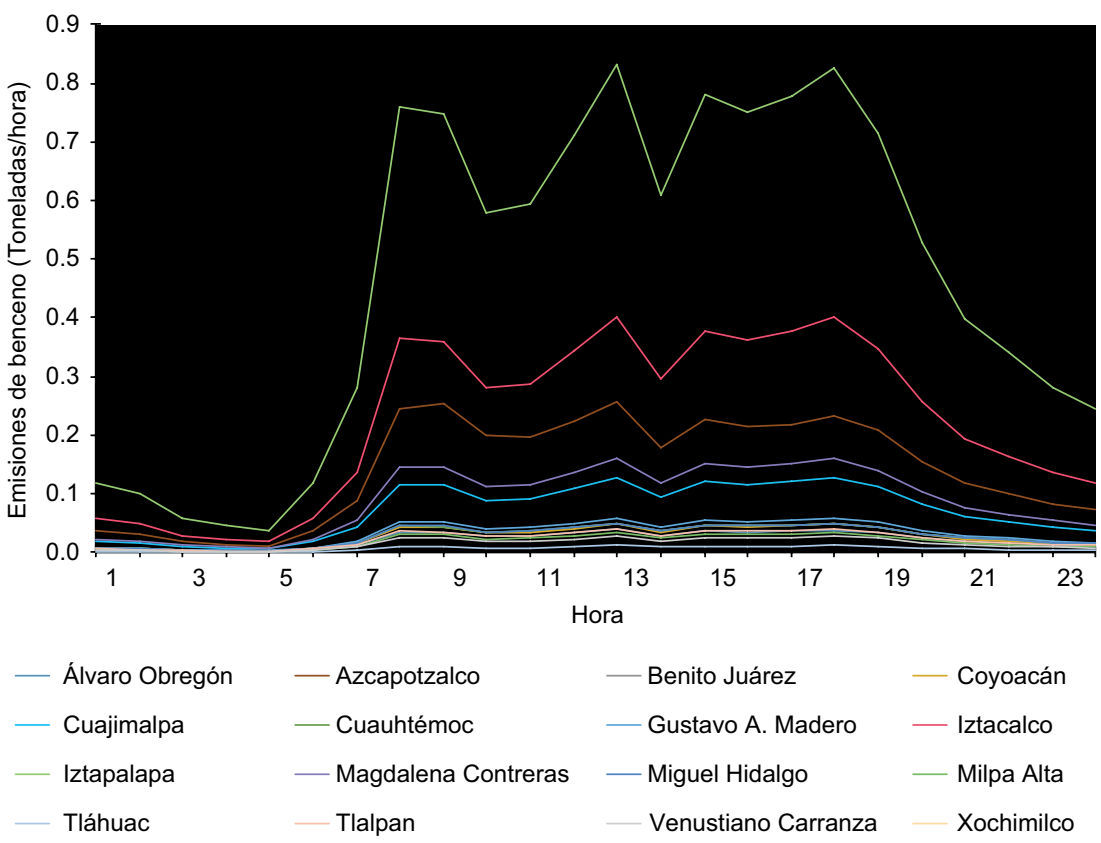

Fig. 8. Emisiones de benceno por hora

Gasolinas Reformuladas Fase 2, establece condiciones regulatorias que obligan a controlar la emisión de compuestos orgánicos volátiles totales en las gasolinas que se utilizan en ciudades con problemas de calidad del aire por ozono. Además, en su evaluación sobre las modificaciones a la citada norma, el INECC detalló que el contenido de $2 \%$ que se permite en las gasolinas nacionales en comparación con el $1 \%$ en las ciudades con alta concentración de ozono en Estados Unidos, produce una mayor contaminación del aire, por lo que recomienda reducir el contenido máximo de este aromático a $1 \%$ en todas las gasolinas nacionales. Por esta razón, la gasolina convencional estadounidense presenta menores emisiones de contaminantes tóxicos que las gasolinas nacionales (INECC 2017).

Algunos de los métodos reportados para la determinación de compuestos aromáticos en las gasolinas incluyen el uso de un cromatógrafo con reflujo (backflush) para una mejor separación (Diehl y Di Sanzo 2005). Otras técnicas de análisis incluyen el uso de un cromatógrafo de gases y la identificación de los aromáticos por medio de espectroscopia y por resonancia magnética nuclear (Singh et al. 2003).

Los métodos de la American Society for Testing and Materials (ASTM) incluidos en la NOM-016CRE-2016 para la determinación del porcentaje en volumen $(\% \mathrm{v})$ del benceno en gasolinas son ASTM D5580, ASTM D6277 y ASTM D3606. Para los métodos D5580-02 (ASTM 2002) y D3606-04a (ASTM
2004) se contempla el uso de un cromatógrafo de gases, y el método D6277-01 (ASTM 2001) se basa en el uso de un equipo de infrarrojo. Sin embargo, existe un método análogo al D3606, el ASTM D5769 (ASTM 2015) que contempla el uso de un cromatógrafo de gases acoplado a un espectrofotómetro de masas (GC/MS). Las diferencias entre los métodos D3606 y D5769 son mínimas, siendo la principal el uso del espectrofotómetro de masas para la identificación de los componentes en el cromatograma con el apoyo de bases de datos existentes.

Con referencia al contenido de benceno en gasolinas, el volumen mínimo para la Magna fue de $0.267 \%$ en la alcaldía Iztacalco y el máximo de $0.545 \%$ en la alcaldía Cuajimalpa de Morelos, mientras que para la gasolina Premium el volumen mínimo de benceno fue de $0.255 \%$ en la alcaldía Coyoacán y el máximo de $0.317 \%$ en la alcaldía Iztacalco. Estas cifras permiten afirmar que las gasolinas evaluadas cumplen con el límite establecido por la NOM-016-CRE-2016 de $1 \%$ en volumen de benceno. De manera general, el contenido de benceno promedio en las gasolinas de la CdMx durante febrero de 2017 fue de $0.361 \%$ en volumen para la Magna y de $0.281 \%$ en volumen para la Premium.

De acuerdo con la solicitud 1867900095316 presentada al INAI se muestran los promedios del contenido de benceno de las gasolinas Magna y Premium producidas por el SNR (el cual está 
constituido por seis refinerías: Cadereyta, Ciudad Madero, Salamanca, Tula, Minatitlán y Salina Cruz) durante el periodo enero-noviembre de 2016 (Fig. 5). Los valores promedio de las medias mensuales del contenido de benceno fueron de $1.288 \%$ en volumen para la Magna y de $0.843 \%$ en volumen para la gasolina Premium. Estos valores se encuentran dentro de la NOM-EM-005-CRE-2015. Especificaciones de calidad de los petrolíferos, la cual establece que el contenido de benceno de las gasolinas Magna y Premium en las zonas metropolitanas del Valle de México, de Guadalajara y de Monterrey debe ser de $1 \%$, y de $2 \%$ en el resto del país; sin embargo, en el caso de la refinería de Minatitlán, el contenido promedio mensual de benceno fue de $2.378 \%$ en volumen, superando el valor permitido por la norma. Asimismo, se observa menor concentración de benceno en las gasolinas importadas, lo que implica que desde el punto de vista de la calidad son mejores que las producidas en el país.

Con referencia a la estimación de emisiones de benceno por automóviles para cada una de las alcaldías de la CdMx, se observa un total de $29565.96 \mathrm{~kg}$ en febrero de 2017. Si se considera este valor como promedio anual, se podría estimar un total de $354.79 \mathrm{t}$ de benceno durante 2017 en la CdMx considerando una flota vehicular de 4421797 unidades; sin embargo, en el Inventario de Emisiones de la CdMx de 2014 (SEDEMA 2016) se indica que una flota vehicular de 1429080 de autos particulares emitió 241.1 t de benceno. Al comparar estas cifras se puede apreciar que el aumento de la flota vehicular induciría una mayor emisión de benceno; sin embargo, debido a que el contenido de benceno en las gasolinas utilizadas en la CdMx es menor al $1 \%$, las emisiones de este compuesto son menores a lo esperado.

Cabe destacar que la mayor emisión de benceno (11 223.09 kg/mes) se presentó en la alcadía Iztapalapa, mientras que la menor (118.62 kg/mes) se presentó en Xochimilco. Estos resultados corresponden con el número de vehículos registrados en ambas delegaciones.

El uso de simuladores para estimar las emisiones de benceno en automóviles permite deducir que la mayor contribución a los procesos de combustión y evaporación ocurre en el arranque por el escape del motor, debido a la mayor cantidad de contaminantes emitidos durante el arranque en frío o durante los minutos que tarda el automóvil en calentarse.

En referencia a las emisiones de benceno a lo largo del día, en las 16 alcaldías de la CdMx se observa variación relacionada con el número de vehículos registrados en cada una de ellas. Además, la emisión más alta de benceno se aprecia entre las 7.00 y las 9:00 horas, así como entre las 13:00 y las 18:00 horas, lo cual coincide con el mayor tránsito vehicular debido a la entrada y salida de las escuelas, así como del horario laboral.

\section{CONCLUSIONES}

$\mathrm{Al}$ evaluar el contenido promedio de benceno en las gasolinas comercializadas en la CdMx durante febrero de 2017 mediante la norma ASTM D5769-15 (ASTM 2015), se encontró que éste fue de $0.361 \%$ en volumen para la gasolina Magna y de $0.281 \%$ en volumen para la gasolina Premium. Estos valores son menores al contenido de benceno en las gasolinas importadas $(0.550 \%$ en volumen) y al de las gasolinas producidas por el SNR $(1.288 \%$ en volumen para la gasolina Magna y $0.843 \%$ en volumen para la gasolina Premium) durante 2016. Es importante considerar estos valores, ya que una mayor cantidad de benceno presente en las gasolinas contribuye a una mayor concentración de este compuesto en el aire.

La estimación experimental del contenido de benceno en la gasolina Magna que se comercializa en la CdMx se utilizó para estimar, con el programa MOVES2014a, las $354.79 \mathrm{t}$ de emisiones de benceno por fuentes móviles (automóviles) para la CdMx durante 2017, y se determinó que la mayor contribución a las emisiones de benceno ocurrió durante el proceso de arranque por el escape del motor. Estas emisiones (354.79 t de benceno/año) aumentaron el $4 \%$ de acuerdo con lo reportado en 2014 (338.6 t de benceno/año) por la SEDEMA. Este valor no fue tan alto debido a que la concentración de benceno en las gasolinas fue menor al $1 \%$ en volumen.

\section{AGRADECIMIENTOS}

Se agradece a la Secretaría de Ciencia, Tecnología e Innovación de la Ciudad de México (SECITI) por los recursos económicos otorgados mediante el Convenio Específico de Colaboración SECITI/054/2016.

\section{REFERENCIAS}

ASTM (2001). D6277-01. Standard test method for determination of benzene in spark-ignition engine fuels using mid infrared spectroscopy. American Society for Testing and Materials International. West Conshohocken, PA, EUA, 10 pp. DOI: 10.1520/D6277-01 
ASTM (2002). ASTM D 5580-02. Standard test method for determination of benzene, toluene, ethylbenzene, $\mathrm{p} / \mathrm{m}$-xylene, o-xilene, $\mathrm{C}_{9}$ and heavier aromatics and total aromatics in finished gasoline by gas chromatography. American Society for Testing and Materials International. West Conshohocken, PA, EUA, 10 pp. DOI: $10.1520 / \mathrm{D} 5580-02$

ASTM (2004). D3606-04a. Standard test method for determination of benzene and toluene in finished motor and aviation gasoline by gas chromatography. American Society for Testing and Materials International. West Conshohocken, PA, EUA, 7 pp.

DOI: $10.1520 / \mathrm{D} 3606-04 \mathrm{~A}$

ASTM (2015). D5769-15. Standard test method for determination of benzene, toluene, and total aromatics in finished gasolines by gas chromatography/mass spectrometry. American Society for Testing and Materials International. West Conshohocken, PA, EUA, 13pp. DOI: $10.1520 / \mathrm{D} 5769-15$

Baklanov A., Molina L.T. y Gauss M. (2016). Megacities, air quality and climate. Atmos. Environ. 126 (2), 235249. DOI: 10.1016/j.atmosenv.2015.11.059

Calderón-Garcidueñas L., Kulesza R.J., Doty R.L., D'Angiulli A. y Torres-Jardón R. (2015) Megacities air pollution problems: Mexico City Metropolitan Area critical issues on the central nervous system pediatric impact. Environ. Res. 137 (2), 157-169.

DOI: 10.1016/j.envres.2014.12.012

CRE (2015). Norma Oficial Mexicana de Emergencia NOM-EM-005-CRE-2015. Especificaciones de calidad de los petrolíferos. Comisión Reguladora de Energía. Diario Oficial de la Federación, 30 de octubre.

CRE (2016). Norma Oficial Mexicana NOM-016CRE-2016. Especificaciones de calidad de los petrolíferos. Comisión Reguladora de Energía. Diario Oficial de la Federación, 29 de agosto.

Diehl J.W. y Di Sanzo F.P. (2005). Determination of aromatics hydrocarbons in gasolines by flow modulated comprehensive two-dimensional gas chromatography. J. Chromatogr. A. 1080 (2), 157-165.

DOI: $10.1016 /$ j.chroma.2004.11.054

Egeghy P.P, Nylander-French L., Gwin K.K., HetzPicciotto I. y Rappaport S.M. (2002). Self-collected breath sampling for monitoring low-level benzene exposures among automobile mechanics. Ann Occup Hyg. 46 (5), 489-500. DOI: 10.1093/annhyg/mef057

INECC (2017). Evaluación de las modificaciones a la NOM-016-CRE-2016. Instituto Nacional de Ecología y Cambio Climático. Guía. Ciudad de México, México, $50 \mathrm{pp}$.

INEGI (2017). Estadísticas de vehículos de motor registrados en circulación. Instituto Nacional de Estadística y Geografía [en línea]. http://www.inegi.org.mx/ sistemas/olap/Proyectos/bd/continuas/transporte/vehiculos.asp? $=$ est $\& \mathrm{c}=13158 \&$ proy $=$ vmrc_vehiculos\# 08/02/18.

Johnson E.S., Langård S. y Lin Y. (2007). A critique of benzene exposure in the general population. Sci. Total Environ. 374 (2-3), 183-198.

DOI: $10.1016 /$ j.scitotenv.2006.11.045

Meraz M., Rodríguez E., Femat R., Echeverría J.C. y Álvarez-Ramírez J. (2015). Statistical persistence of air pollutants $\left(\mathrm{O}_{3}, \mathrm{SO}_{2}, \mathrm{NO}_{2}\right.$ and $\left.\mathrm{PM}_{10}\right)$ in Mexico City. Physica A. 427 (6), 202-217.

DOI: $10.1016 /$ j.physa.2015.02.009

Molina M.J. y Molina L.T. (2004) Improving air quality in megacities: Mexico City case study. Ann. N.Y. Acad. Sci. 1023, 142-58. DOI: 10.1196/annals.1319.006

Moro A.M., Brucker N., Charão M.F., Baierle M., Sauer E., Gothel G., Barth A., Nascimento S.N., Gauer B., Durgante J., Amaral B.S., Neto F.R.A., Gioda A. y Garcia S.C. (2017). Biomonitoring of gasoline station attendants exposed to benzene: Effect of gender. Mutat. Res. 813 (1), 1-9.

DOI: 10.1016/j.mrgentox.2016.11.002

PEMEX (2017). Guía Pemex [en línea]. http://guiapemex. pemex.com/SitePages/home.aspx 20/03/2017.

Perry R. y Gee I.L. (1994). Vehicle emissions and effects on air quality: Indoors and outdoors. Indoor Built Environ. 3 (4), 224-236. DOI: 10.1177/1420326X9400300409

SEDEMA (2013). Inventario de emisiones de contaminantes y de efecto invernadero 2012. Secretaría del Medio Ambiente del Gobierno de la Ciudad de México. Informe. Distrito Federal, México, 122 pp.

SEDEMA (2016). Inventario de emisiones de la CdMx 2014. Contaminantes criterio, tóxicos y de efecto invernadero. Secretaría del Medio Ambiente de del Gobierno de la Ciudad de México. Informe. Ciudad de México, México, 134 pp.

SEMARNAT (2006). Norma Oficial Mexicana NOM086-SEMARNAT-SENER-SCFI-2005. Especificaciones de los combustibles fósiles para la protección ambiental. Secretaría del Medio Ambiente y Recursos Naturales. Diario Oficial de la Federación, 30 de enero.

Singh A.P., Mukherji S., Tewari A.K., Kalsi W.R. y Sarpal A.S. (2003). Determination of benzene and total aromatics in commercial gasolines using packed column GC and NMR techniques. Fuel 82 (1), 23-33.

DOI: 10.1016/S0016-2361(02)00157-6

Torres M. (2016). Franquicia Pemex [en línea]. http:// www.franquiciapemex.com/octanaje/21 regi.html 15/11/2016.

Verma D.K. y des Tombe K. (2002). Benzene in gasoline and crude oil: occupational and environmental implications. AIHA Journal 63 (2), 225-30. 
Wallace L.A., Pellizari E.D., Hartwell T.D., Sparacino C., Whitmore R., Sheldon L., Zelon H. y Perritt R. (1987). The TEAM (Total Exposure Assessment Methodology) study: personal exposures to toxic substances in air, drinking water, and breath of 400 resident of New Jersey, North Carolina, and North Dakota. Environ Res. 43 (2), 290-307. DOI: 10.1016/S0013-9351(87)80030-0
Wallace L. (1996). Environmental exposure to benzene: An update. Environ. Health Perspect. 104 (6), 1129-1136. DOI: $10.1289 /$ ehp. 961041129 